\title{
Diffusion in magnetic fields
}

\author{
G. Alecian \\ LUTH, Observatoire de Paris-Meudon, F-92195 Meudon Cedex, France \\ email: georges.alecian@obspm.fr
}

\begin{abstract}
Diffusion of elements in a stellar plasma is strongly modified by the presence of magnetic fields for two primary reasons. The first is that the average motions of ions in outer atmospheres are, because of their charge, substantially constrained by the magnetic field. Both its intensity and orientation play a role. The second is the Zeeman desaturation of absorption lines that often produces amplifications of the radiative accelerations. These effects are important and must lead to the building of complex surface abundance structures. I will present how these two effects are generally modeled and what results have, up to now, been obtained. Future developments will also be considered.
\end{abstract}

Keywords. Diffusion, stars: abundances, stars: atmospheres, stars: magnetic fields

\section{Introduction}

When one speaks about the diffusion of elements in stars, one designates the macroscopic consequences of a microscopic process which involves a large number of erratic motions of charged particles. Therefore, the presence of a magnetic field has obviously to be taken into account in the description of that process. An approximate theory has been given by Chapman \& Cowling (1970). A typical effect of the magnetic fields on the diffusion velocity of metals in the atmospheres of magnetic Ap stars was first discussed by Vauclair, Hardorp \& Peterson (1979) for the case of Si accumulation. I will present some aspects of diffusion across magnetic lines in Sec. 2.

There is another effect which was often neglected: the Zeeman desaturation of absorption lines. Among the important terms in the equation of the diffusion velocity, there is the radiative acceleration which has a particular status: it depends strongly on the atomic properties (transitions) of diffusing species and also on the ionic concentrations. One reason why the concentration of a given ion is involved is that the saturation of absorption lines determines the efficiency of the momentum transfered from radiation field to the considered species. One then understands how the Zeeman effect can influence the radiative accelerations and in turn the diffusion process itself. This effect was often neglected because in the few studies which were done, Zeeman desaturation was found to modify radiative accelerations by a smaller amount than their expected accuracies. The numerical cost to compute it appeared too high with regard to the benefits, and large atomic databanks were not available at that time. These considerations are no longer valid. After the pioneering work of Babel \& Michaud (1991b), the importance of the Zeeman desaturation has been confirmed by the detailed study and the extensive numerical computations of Alecian \& Stift (2004). I will discuss Zeeman amplification in Sec. 3.

Of course, magnetic fields can affect element stratifications in stars in many other ways, for instance through interactions with macroscopic motions of the plasma (see also Leblanc, Michaud \& Babel (1994)) who have considered the effect of Lorentz forces on model atmospheres). We consider in this talk only the effects of the magnetic fields on the microscopic diffusion velocities and the radiative accelerations. Some new results on 
diffusion in magnetic fields, with both effects (particle motions and Zeeman desaturation) will be shown in Sec. 4.

\section{Diffusion velocity}

To model diffusion processes in Ap stellar atmospheres (Michaud 1970), one needs to compute the diffusion flux for each element. This flux is obtained through a weighted sum of the diffusion velocities corresponding to the various ionisation states (including neutral) for each species considered. We do not detail here how to obtain these velocities, rather we limit ourselves to the effects of the magnetic field.

\subsection{Diffusion across horizontal magnetic lines}

The diffusion velocity of a given type of particles in a partially ionised plasma is the average quantity which remains after the summation of a large number of microscopic movements of particles. These movements are unaffected by the magnetic field when particles are neutral. But charged particles have a circular motion in the direction transverse to the magnetic field. The resultant motion during the time between two collisions is a spiral along magnetic lines, but the diffusion velocity remains rectilinear. An approximate theory of diffusion in magnetic fields can be found in Chapman \& Cowling (1970). These authors have shown that the diffusion velocity of charged particles, when it is orthogonal to magnetic lines, is reduced by the factor:

$$
f_{\text {slow }, i}=\left(1+\omega_{i}^{2} t_{i}^{2}\right)^{-1}
$$

where $t_{i}$ is the collision time (the time needed to a particle $i$ with mass $m_{i}$ and charge $Z e$, to deviate through collisions by $\frac{\pi}{2}$ from its initial motion), $\omega_{i} / 2 \pi$ is the cyclotron frequency in a field with intensity $H$ :

$$
\omega_{i}=\frac{Z e H}{m_{i} c}
$$

The average diffusion velocity of an element can be approximated by the following expression (the sums are over the ionisation states $i$ ):

$$
V_{\mathrm{D}} \approx \frac{\sum_{i} N_{i} f_{\text {slow }, i} V_{\mathrm{D} i}}{\sum_{i} N_{i}}
$$

This formula was used by Vauclair, Hardorp \& Peterson (1979) to study the Si accumulation in magnetic Ap stars. Silicon is found to be strongly overabundant in magnetic Ap stars and more or less normal in nonmagnetic peculiar stars. Vauclair, Hardorp \& Peterson (1979) have shown that the radiative acceleration on ionized Si is not very strong and cannot lead to high overabundances, even if the acceleration of the neutral state is large. This explains the quasi-normal abundance of Si in the nonmagnetic stars. But in the magnetic Ap stars, the downward diffusion flux due to the settling of Si ions is slowed down by the magnetic field (small $f_{\text {slow }, i}$ in Eq.(2.1)), while for neutral state, $f_{\text {slow }, 0}=1$ ensures a positive velocity $V_{D}$ and leads to Si overabundances. These authors concluded that their diffusion model is compatible with the Si abundances observed in peculiar stars. This study has been extended to the oblique rotator model by Michaud, Megessier \& Charland (1981) assuming a dipole plus quadrupole magnetic structure. They have shown how elements could accumulate according to the orientation of the magnetic field (at the magnetic poles and/or the magnetic equator). 


\subsection{Oblique magnetic lines}

In the previous section, we have essentially considered the average diffusion velocity across horizontal magnetic lines. At the surface of magnetic stars the direction of the magnetic field lines varies from 0 to $\frac{\pi}{2}$, between magnetic poles(s) and equator(s). This angular dependence contributes to explaining the inhomogeneous abundance distribution over the stellar surface: the sensitivity of the diffusion velocity to the inclination of the magnetic lines determines the size of abundance structures [see Michaud, Megessier \& Charland (1981), and Alecian \& Vauclair (1981)]. In oblique magnetic fields lines, the diffusion velocity vector is no longer vertical, rather its inclination depends on the angle $\theta$ of the magnetic lines to the vertical according to the expressions given by Alecian \& Vauclair (1981). The vertical ( $Z$ axis) component is:

$$
V_{H, Z i} \approx V_{i}\left(f_{\text {slow }, i}+\frac{1}{f_{\text {slow }, i}} \cos ^{2} \theta\right),
$$

and the horizontal one $(X$ axis $)$ is:

$$
V_{H, X i} \approx \frac{f_{\text {slow }, i} V_{i}}{2} \sin 2 \theta
$$

$V_{i}$ is the diffusion velocity for zero magnetic field.

The horizontal component given by expression (2.5), represents an horizontal diffusion of ions only. The diffusion velocity of the element will be affected through the averaged velocity (2.3). Does this means that horizontal diffusion could play a role for surface abundance structures observed in magnetic Ap stars (see for instance Kochukhov et al. (2004))? The answer is no! In the case of silicon, Megessier (1984) has estimated the time scale for Si migration from the pole to the equator. This time scale appears to be of the order of $10^{7}$ years! This is smaller than the lifetime of Ap stars on the main sequence. However, it implies that the lifetime of the magnetic structures should be also of the same order of magnitude if one would hope to have an observable effect due to horizontal diffusion. On the other hand, and this is more constricting, the time scales of vertical diffusion are much smaller ( 4 or 5 orders of magnitude smaller), because vertical height scales are much smaller too. Vertical particle diffusion flux dominates. One can then conclude that its seems rather unlikely that horizontal diffusion may have a significant effect. This means that the abundance inhomogeneities on magnetic Ap stars (patches or rings) are mainly due to the angular dependence of the vertical component of the velocity vector as shown in Eq.(2.4), combined with the local mass-loss velocity.

\section{Radiative acceleration}

A pioneering work, based both on analytical approximations to the polarised radiative transfer problem and on numerical solutions, was carried out by Babel \& Michaud (1991a). More recently, Alecian \& Stift (2004) have investigated the same problem in detail by numerical means. Their exhaustive computations were carried out for 329 ions, using the VALD atomic database (Piskunov et al. 1995), solving numerically the polarised radiative transfer equation by means of a new formulation of the Zeeman Feautrier method (Auer, Heasley, \& House 1977, Rees, Murphy, \& Durrant 1989) includes magnetooptical effects, and provides for the correct treatment of line blending.

The radiative acceleration is a vector which usually is not more vertical when a magnetic field is present. It is obtained through the following integral over the solid angle $\Omega$ 


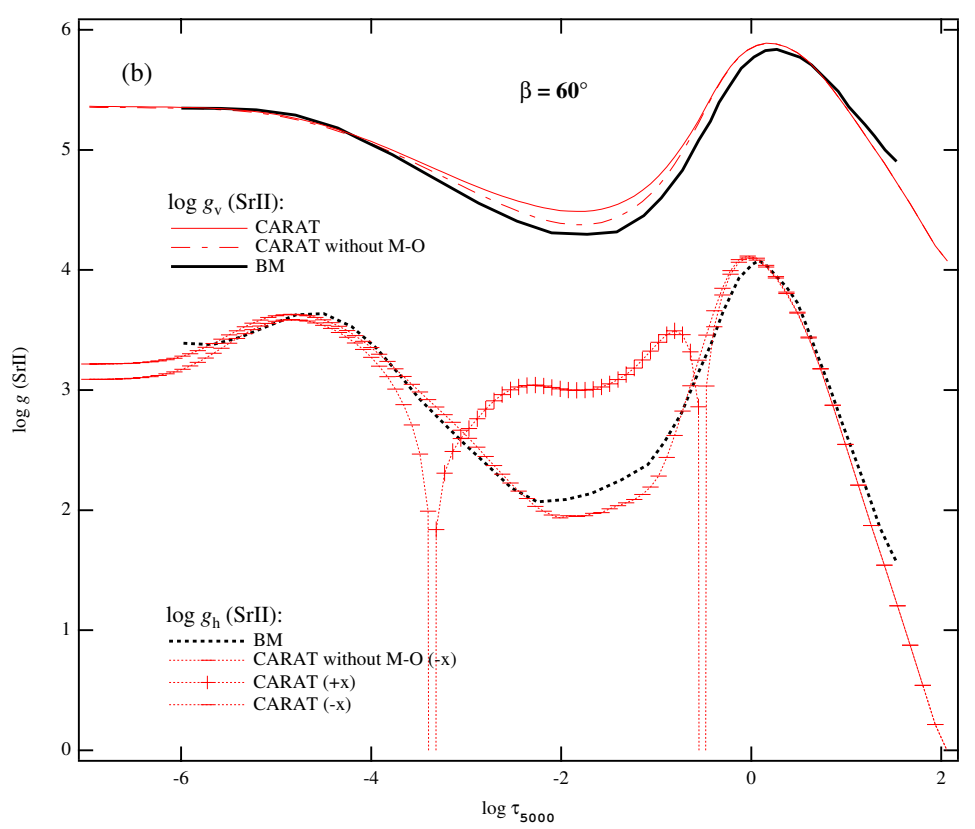

Figure 1. Radiative accelerations due to the Sr II $\lambda 4077$ line in the non-magnetic case and in a $1.5 \mathrm{~T}$ field, based on a $8500 \mathrm{~K}, \log g=4.0$ ATLAS9 (Kurucz 1993) model atmosphere (from Alecian \& Stift 2004). This plot shows a comparison between the accelerations obtained by Babel \& Michaud (1991a)(BM) and results of CARAT code, both with and without magneto-optical effects. The field is vertical. The vertical acceleration is shown by the upper curves and the horizontal one by the lower curves. The field is inclined by $60^{\circ}$ with respect to the vertical. Note that the horizontal acceleration changes sign twice over optical depth when m.-o. effects are taken into account.

and frequency $\nu$ (from Alecian \& Stift 2004):

$$
\mathbf{g}_{i}^{\mathrm{rad}}=\sum_{k, m>k} \frac{n_{i, k}}{n_{i} A m_{\mathrm{p}} c} \iint(\mathbf{e} \cdot \mathbf{I}) \boldsymbol{\Omega} d \Omega d \nu
$$

- with $n_{i}$ the total number density $\left(\mathrm{cm}^{-3}\right)$ of ions $A^{+i}, n_{i k}$ the number density of ions in initial lower level $k$,

- and the summation extending over all transitions from initial level $k$ to higher levels $m$.

- $A m_{\mathrm{p}}$ is the mass $(\mathrm{g})$ of the ion,

- $(\mathbf{e} \cdot \mathbf{I})$ denotes the inner product of the vector $\mathbf{e}=\kappa_{\circ}\left\{\phi_{I}, \phi_{Q}, \phi_{U}, \phi_{V}\right\}$ (line absorption matrix elements, see Alecian \& Stift (2004) for details), with the Stokes vector I.

\subsection{Zeeman amplification}

The results presented here have been obtained by Alecian \& Stift (2004) with their new LTE diffusion code CARAT (Code pour les Accélérations Radiatives dans les ATmosphères) that solves the polarized radiation transfer equations for Eq.(3.1) on massively parallel multiprocessor machines.

A comparison of a result obtained by CARAT with the curves of Babel \& Michaud (1991a) is shown in Fig. 1. The role of magneto-optical effect is highlighted and also the particular behavior of the horizontal component of the radiative acceleration. This later appears negligible compared to the vertical component, at least for the cases Alecian \& Stift (2004) have considered (see their paper for more details). 


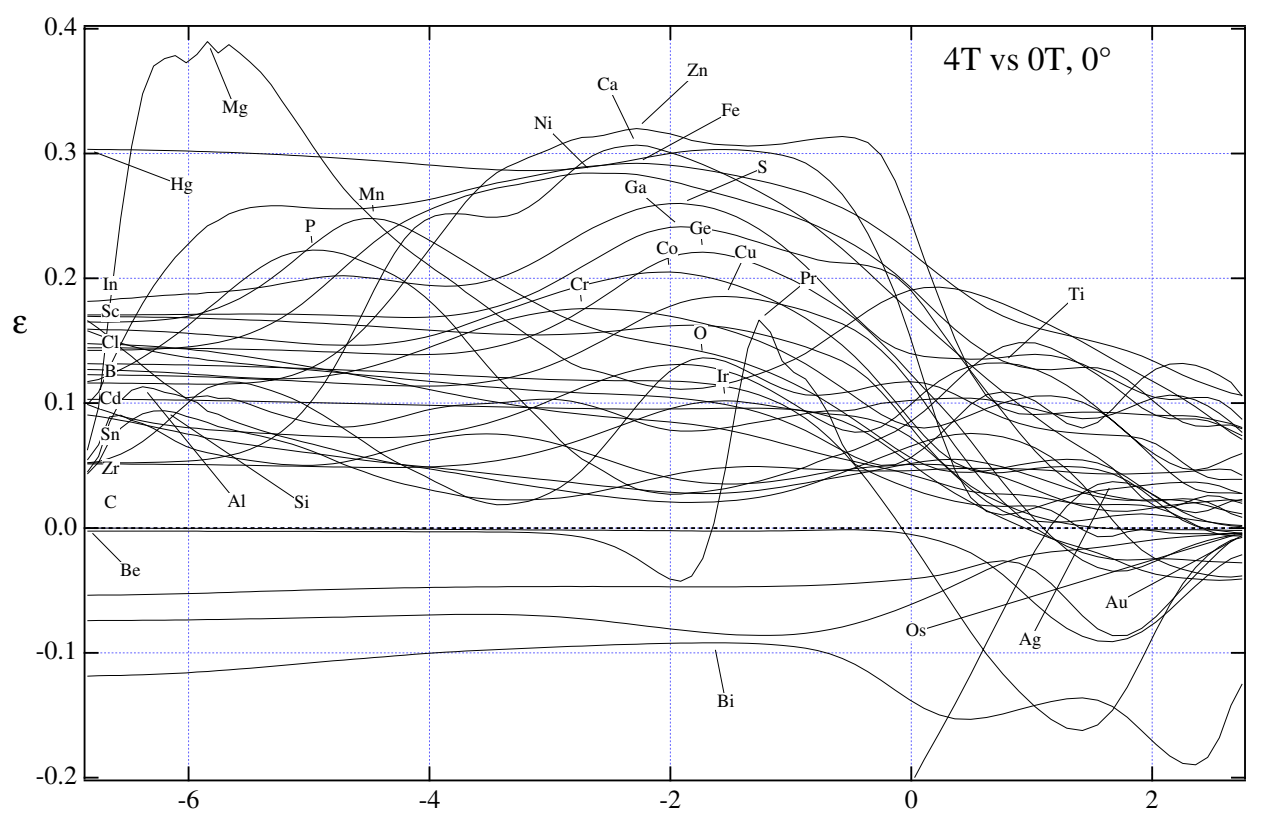

$\log \tau_{5000}$

Figure 2. Amplification of the radiative accelerations of the chemical elements due to Zeeman splitting as a function of optical depth and of the magnetic field strength (from Alecian \& Stift 2004). Logarithmic amplifications are displayed for a field strength of $4 \mathrm{~T}$ for the vertical magnetic lines. Curves are plotted only when $|\varepsilon|>0.0414$ dex (10\% amplification) at any depth point.

Some amplifications are shown in Fig. 2. They have been computed for a $T_{\text {eff }}=12000 \mathrm{~K}$ and $\log g=4.0$ ATLAS9 (Kurucz 1993 ) model atmosphere. Amplifications are based on the simplified expression for the total radiative accelerations $g^{\text {rad }}$ given by a sum of ion accererations (3.1), weighted by their relative population. The magnetic field is vertical. The amplifications $\left(\varepsilon=\log g^{\mathrm{rad}}\right.$ (magnetic) $-\log g^{\mathrm{rad}}$ (nonmagnetic) $)$ are at most about 0.40 dex for $4 \mathrm{~T}$. This corresponds to a maximum increase of radiative accelerations by a factor 2.5. This increase is by no means negligible.

\section{Preliminary results on diffusion in magnetic Ap stars}

As emphasized above, the study of microscopic diffusion of elements in magnetic fields needs to compute the vertical components (Eq. 2.4) of the diffusion velocities of ions, using the radiative acceleration given by Eq. (3.1). Actually, one of the main goals of Ap stars modelling is to know what abundance stratification can be obtained. Unfortunately, this is beyond present abilities because the stratification process is non-linear and timedependent, and needs much more computing power than presently available as well as theoretical and software developments. However, a first guess of what stratifications could be expected can be obtained by considering the fluxes of the diffusing species at the beginning of the process (when the elements have still their normal solar abundances and when no competing processes are considered). This kind of study was first carried out by Alecian \& Vauclair (1981) who neglected the Zeeman effect. A preliminary detailed 


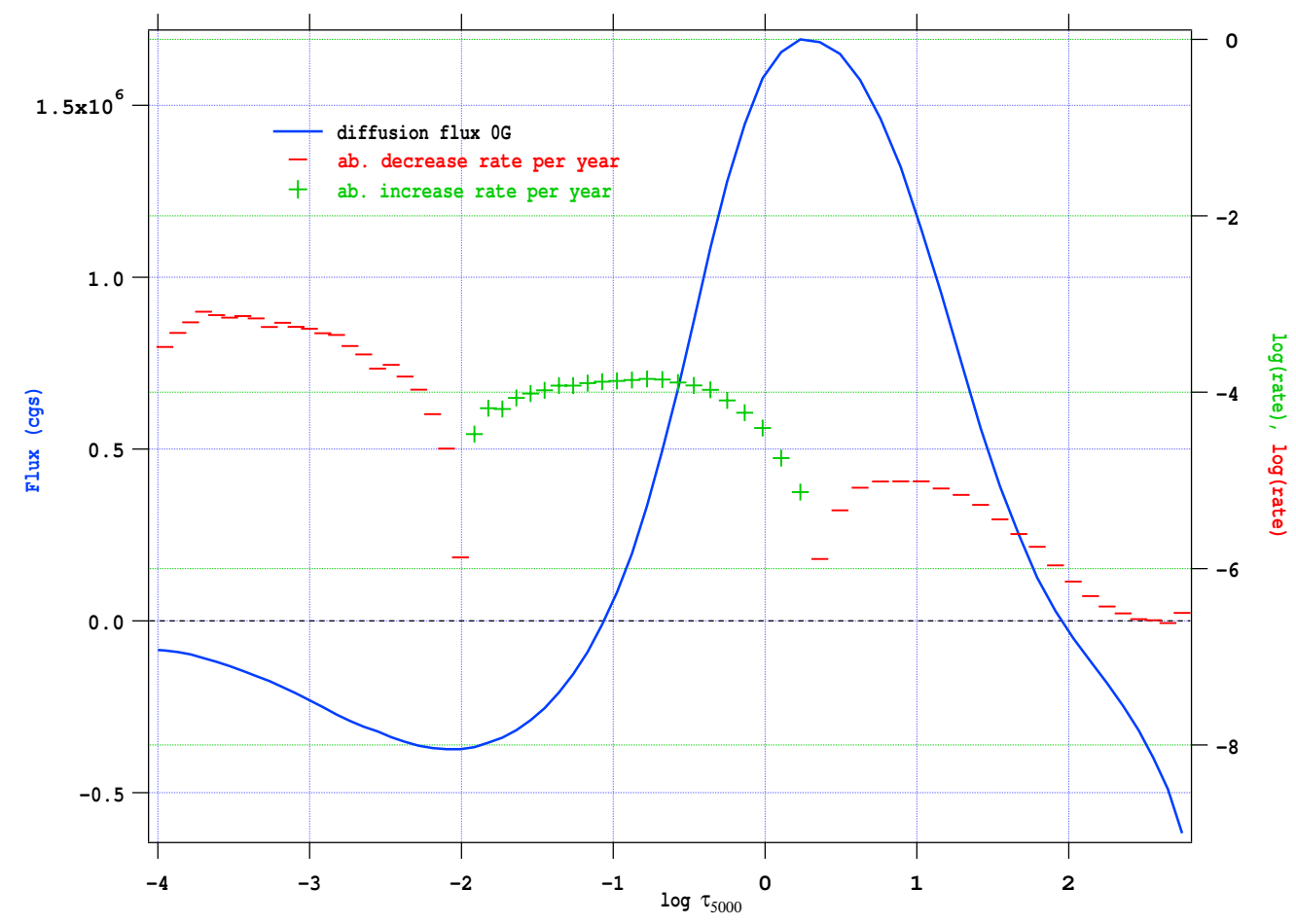

Figure 3. Diffusion flux of Al (left axis) for zero magnetic field (for solar abundance). The logarithm of the abundance change rate per year is also shown (right axis). The + curve indicates abundance increase, and the - one the abundance decrease.

calculations of aluminum fluxes (Alecian \& Stift 2005) are shown in Fig. 3 and Fig. 4 in the case of the $T_{\text {eff }}=12000 \mathrm{~K}$ model atmosphere noted previously.

For zero magnetic field (Fig. 3), aluminum is pushed upwards in the atmosphere in layers with $-1<\log \tau_{5000}<+2$. This allows us to predict (curves with symbols + and - ) that the abundance of $\mathrm{Al}$ will start to increase in layers with $-2<\log \tau_{5000}<+0.2$ by -4 dex per year (the abundance of $\mathrm{Al}$ will be doubled in about $10^{4}$ years if one neglects the non-linearity of the process). This increase rate is rather small compared to that of many iron peak elements. The effect of a magnetic field (1 Tesla) with various inclinations are shown in Fig. 4. The difference between the $0 \mathrm{~T}$ and $1 \mathrm{~T}$ (vertical) fluxes is due to the modest Zeeman amplification of $\mathrm{Al}$ radiative acceleration (these two curves would be superimposed if Zeeman effect were neglected). When the magnetic field is horizontal, the flux is significately reduced as one goes up in the atmosphere. This is due to the increase of the collision time $t_{i}$ which allows charged particles to spiral more lengthly around magnetic field lines.

\section{Conclusions}

We have presented here a short overview of the main effects of magnetic fields on microscopic diffusion. One can reasonably expect that in the short term, one should be able to compute the trends of abundance stratifications in magnetic atmospheres, assuming LTE and assuming that equilibrium stratifications can exit (this is still unknown). Similar modelling is already in progress for the case without a magnetic field (see LeBlanc \& Monin 2005). In the magnetic case, the next steps should be to address self-consistent 


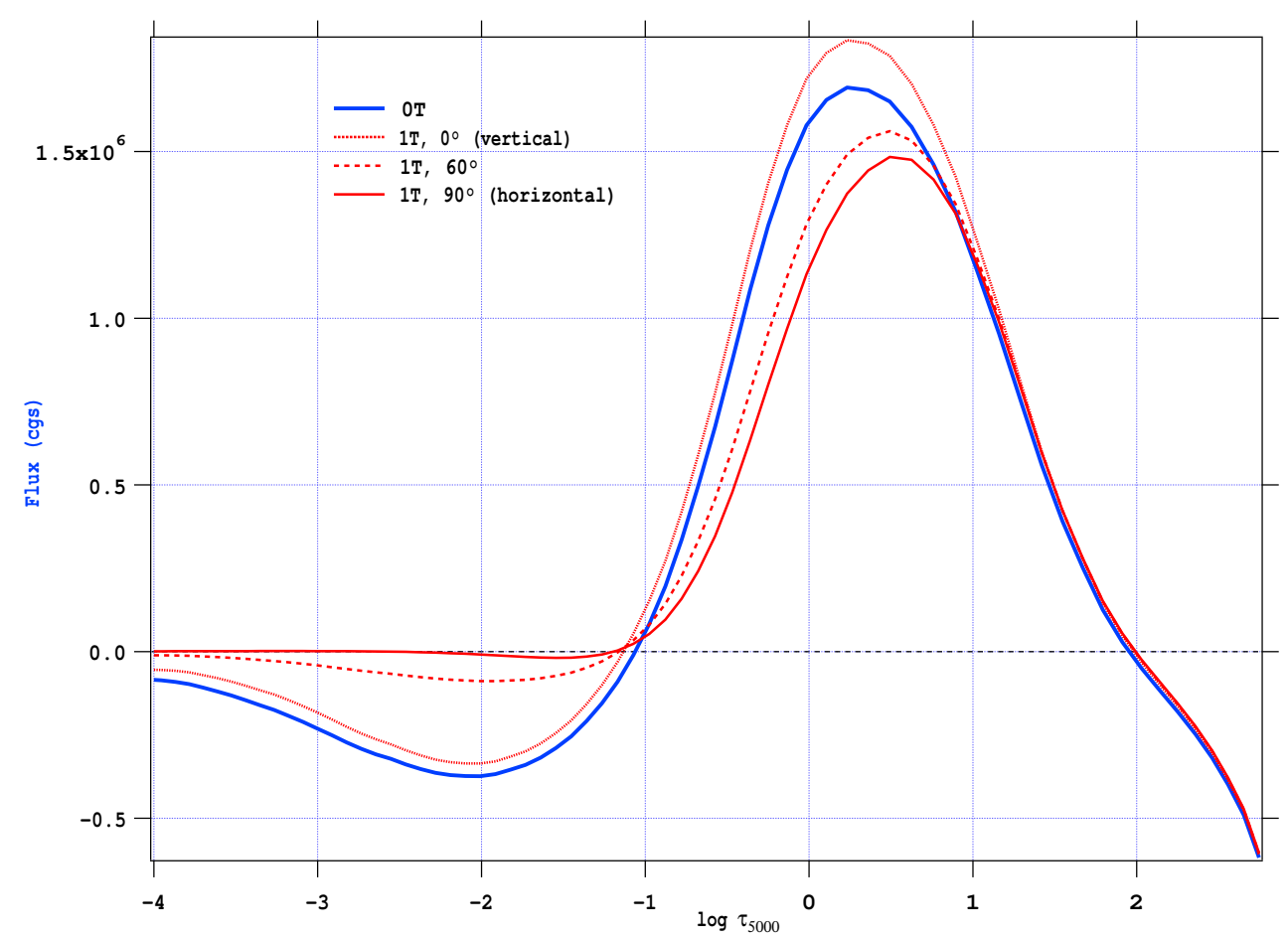

Figure 4. Effect of a magnetic field (1 Tesla) on the diffusion flux of $\mathrm{Al}$ is shown for various inclinations of the magnetic lines. The "OT" heavy solid curve is the same as the one of Fig. 3

modelling (feedback of stratifications on the model atmospheres), NLTE, 2D oblique rotator modelling, and take into account additional physical processes such as ambipolar diffusion, hydrodynamics, etc.

Thus many investigations remain to be done before reaching the 'mythical' goal of a realistic time-dependent numerical simulation of the abundance stratification process.

\section{Acknowledgements}

I thank Martin J. Stift who provided me in time with a new version of the CARAT code (Alecian \& Stift 2005) which has contributed to the computation of the diffusion fluxes shown in Sec. 4. His work has the support of the Austrian Science Fund (FWF), project P16003-N05 "Radiation driven diffusion in magnetic stellar atmospheres".

\section{References}

Alecian G., Artru M.-C., 1987, A\&A, 186, 223

Alecian G., Stift M.J., 2004, A\&A, 416, 703

Alecian G., Stift M.J., 2005, in preparation

Alecian G., Vauclair S., 1981, A\&A, 101, 16

Auer, L.H., Heasley, J.N., \& House, L.L. 1977, ApJ, 216, 531

Babel J., Michaud G., 1991a, A\&A, 241, 493

Babel J., Michaud G., 1991b, A\&A, 248, 155

Chapman S., Cowling T.G., 1970, The Mathematical Theory of Non-uniform Gases, Cambridge University Press, 3rd ed.

Hui Bon Hoa A., Alecian G., Artru M.C., 1996, A\&A, 313, 624 
Kochukhov, O., Bagnulo, S., Wade, G.A., Sangalli, L., Piskunov, N., Landstreet, J.D., Petit, P. \& Sigut, T.A.A., 2004, A\&A, 414, 613

Kurucz, R. 1993, CDROM Model Distribution, Smithsonian Astrophys. Obs.

Kuschnig, R., Ryabchikova, T.A., Piskunov, N.E., Weiss, W.W., Gelbmann, M.J. 1999, A\&A, 348,924

Leblanc F., Michaud G., Babel J., 1994, ApJ, 431, 388

LeBlanc F., Monin D. 2005 These Proceedings, 193

Megessier C., 1984, A\&A, 138, 267

Michaud G., 1970, ApJ, 160, 641

Michaud G., Megessier C., Charland Y., 1981, A\&A, 103, 244

Piskunov, N.E., Kupka, F., Ryabchikova, T.A.,Weiss, W.W., \& Jeffery, C.S. 1995, A\&AS, 112, 525

Rees, D.E., Murphy, G.A., \& Durrant C.J., 1989, ApJ, 339, 1093

Vauclair S., Hardorp J., Peterson D.M., 1979, ApJ, 227, 526

\section{Discussion}

Piskunov: Rapidly oscillating Ap stars show element stratifications at very high atmospheric layers $\left(\tau<10^{-8}\right)$. Can your procedure handle such conditions and what should be done to compute magnetic diffusion in these conditions?

Alecian: As I mentioned, the CARAT code uses LTE physics. Therefore its validity domain is limited to layers with $\tau>10^{-5}$ or $10^{-4}$. On the other hand, the magnetic field is a very strong barrier in high atmospheric layers. Then the computation of diffusion velocities at such high layers $\left(\tau<10^{-8}\right)$ in strong magnetic fields using the same approximations as in the lower atmosphere is probably not justified.

KuBÁt: What is the role of elastic collisions in your model? How are they included into your calculations?

Alecian: Elastic collisions are one of the hypotheses imposed to establish the usual equations for diffusion velocities in the framework of the kinetic theory of gases (see Chapman \& Cowling 1970). The diffusion coefficients we use are determined assuming elastic collisions.

DwORETSKY: In HgMn stars the observed range of iron abundances is nearly two orders of magnitude. Are these more likely to be equilibrium abundances or "snapshots" in which Fe abundances evolve with time in a complicated way?

Alecian: The building of abundance stratifications by diffusion is a non-linear process and some instabilities might occur. In my opinion, clouds of metals in optically thin layers could disappear and form again. Therefore, the abundance scatter that you mention should not correspond to any equilibrium abundances. 